\title{
Characterization of Staphylococcus aureus infections among children in an outpatient clinic, China
}

\author{
Huifen Ye, Junshao Zeng, Wenzhou Qin, Yi Feng, Haitao Tan*
}

Guigang People's Hospital, Guangxi, China

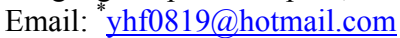

Received 20 May 2013; revised 20 June 2013; accepted 28 June 2013

Copyright (C) 2013 Huifen Ye et al. This is an open access article distributed under the Creative Commons Attribution License, which permits unrestricted use, distribution, and reproduction in any medium, provided the original work is properly cited.

\begin{abstract}
Objective: We aimed to investigate the epidemiology and identify antibiotic resistance patterns of isolates from children with skin and soft tissue infections attending an outpatient clinic in Southern China. Method: An observational study of outpatient pediatric patients was conducted in a rural area of Guangxi. Infections were characterized in 230 patients and staphylococcal isolates tested for susceptibility to a range of antibiotics. Results: Among the 307 patients, $38.5 \%$ were infants. Culture yielded Staphylococcus aureus (S. aureus) in 230 patients, of which $24(10.4 \%)$ were methicillin-resistant $S$. aureus (MRSA). Staphylococcal strains were most isolated from hand, foot and umbilicus. The 1 - 12 mon group had the highest staphylococcal infection rate ( $86 \%)$, followed by the 6 - 12 year group, but MRSA was more common in the older children (19.5\% of $S$. aureus). MRSA had significantly more resistance than MSSA to chloromycin (46\% vs $11 \%)$, clindamycin ( $67 \%$ vs $19 \%)$, gentamicin ( $33 \%$ vs $2 \%$ ), rifampicin ( $25 \%$ vs $2.9 \%$ ), and sulphamthoxazole-trimethoprim (17\% vs $3 \%)$. Conclusion: $S$. aureus remains a leading cause of pediatric skin and soft tissue infections. Over $10 \%$ of isolates were methicillin-resistant with high rates of resistance to non-beta lactam antibiotics, reducing options for therapy and limiting choices for empirical treatment.
\end{abstract}

Keywords: Skin and Soft Tissue Infections; Community-Acquired Methicillin-Resistant

Staphylococcus aureus; Pediatrics

\section{INTRODUCTION}

Staphylococcus aureus (SA) is an important pathogen

"Corresponding author. causing infections ranging from superficial skin infections to life threatening conditions [1]. Methicillin-resistant S. aureus (MRSA) evolved in healthcare facilities causing nosocomial infections in both adults and children, but since first reports of community-associated MRSA (CA-MRSA) in children without predisposing risk in 1998 [2], its prevalence continues to increase. The proportion of CA-MRSA isolated from skin and soft tissue infections (SSI) worldwide ranges from $65 \%$ to $91 \%$ [3-5].

CA-MRSA harbors the mecA gene, located in the staphylococcal cassette chromosome mec (SCCmec), which codes for an altered penicillin binding protein, 2a (PBP2a), that decreases affinity for beta-lactam antibiotics [6]. Compared to hospital-associated MRSA, which contains the larger SCCmec elements I, II and III, the SCCmec types present in CA-MRSA are the smaller types IV and V [1]. The smaller elements often do not harbor additional resistance determinants. Panton-Valentin Leukocidin (PVL) toxin is an important virulence factor in CA-MRSA involved in SSI [7]. Children are prone to staphylococcal infections including impetigo, cellulitis and abscesses, and attendance at childcare facilities increases infection risk [8].

MRSA infections in children have been reported, but there is limited data on such infections from China, especially from outside of the major metropolitan areas. This study aimed to investigate the epidemiology of pediatric STIs in attendees at an outpatient clinic in Southern China and determine the antibiotic resistance profiles of $S$. aureus isolates.

\section{METHODS}

Ethical Statement: Written informed consents were signed by parents or supervisors of the pediatric participants, and study protocols were reviewed and approved by the Research Ethical Committee at the Guigang People's Hospital, China. 
Study Population: A prospective observational study was conducted between January 1, 2009 and December 31, 2010. All pediatrics patients (age $\leq 18 \mathrm{yr}, \mathrm{n}=307$ ) attending the hospital outpatient clinic and diagnosed with SSI were included.

Bacterial Strains and Identification of $S$. aureus: Lesions were sampled using sterile cotton-tip swabs premoistened with saline, which were cultured on 5\% sheep blood agar and incubated at $35^{\circ} \mathrm{C}$ for 18 to 24 hours. $S$. aureus was identified by colony morphology, gram stain, catalase production and tube aggregation, followed by a positive result of Slidex ${ }^{\circledR}$ Staph s aggregation test (Biomeriux, France). A cefoxitin disk was used to test for methicillin resistance [9].

Antibiotics Susceptibility: Susceptibility was determined for a range of antibiotics by disc diffusion [9]. E-test was used to determine vancomycin susceptibility with a minimum inhibitory concentration of $\geq 2 \mathrm{mg} / \mathrm{L}$ indicating reduced susceptibility. The inducible clindamycin test (D-test) was used to investigate resistance to clindamycin [9].

Statistics: Data were analyzed using statistical package of social science for windows (SPSS 17.0, SPSS Inc, Chicago). Chi square test was performed to determine difference of $S$. aureus and MRSA infection rates between age groups and seasonal distribution, as well as antibiotic resistance patterns. A $p$ value of 0.05 or below was considered as significant.

\section{RESULTS}

A total of 307 pediatric patients were recruited during the study period. Overall, 230 patients had S. aureus infections $(75.2 \%)$, including 24 caused by MRSA (10.4\%). The most common infected sites by $S$. aureus were hand $(90.0 \%)$, foot $(87.0 \%)$ and umbilicus $(84.8 \%)$, but MRSA were most isolated from foot (20\%), hand (18.5\%), back and lower limb (14.3\%) (Table 1).

Among all the participant subjects in the study population, over one third $(\mathrm{n}=118)$ were infants $(<1$ year $)$, and male patients $(\mathrm{n}=171)$ accounted for $55.9 \%$. The highest S. aureus infection rate was in the $1-12$ mon group $(86.3 \%)$, followed by the $6-12$ yr group $(80.4 \%)$. The latter group had the highest percentage MRSA infections (19.5\%). The percentage of infections caused by MRSA and the overall infection rates differed significantly between age groups $(p<0.05)$. The demographic characteristics are summarized in Table 2.

Over the two year study period, it was found that the highest $S$. aureus and MRSA infection rate, which had reached $85.3 \%$ and $13.6 \%$, occurred in the summer and the rates in winter were $62.7 \%$ and $8.1 \%$. Rates of $S$. aureus and MRSA were significant different between seasons (Table 3).

MRSA strains showed high rates of resistance to erythromycin, clindamycin and chloromycetin. These rates and those of gentamicin, rifampicin and trimethoprim-sulphamethoxazole (TMP-SXT) differed significantly from those of MSSA (Table 4). No resistance to tetracycline or vancomycin was observed.

\section{DISCUSSION}

This study of pediatric outpatient infections was conducted in a rural area of Guangxi Province in Western China, about $150 \mathrm{~km}$ from the provincial capital, Nanning. Living conditions are not as developed as in the major cities. Elsewhere it has been reported that MRSA colonization is associated with low socioeconomic status, inadequate medical insurance, poor access to healthcare and household crowding [10]. However, this has not been well established for pediatric populations. In this present study, children in poor and remote villages have a higher S. aureus infection rate than those live in town or city.

Table 1. S. aureus and MRSA infected sites in children with SSTIs.

\begin{tabular}{cccccc}
\hline Infected sites & Infected cases (n) & MRSA (n) & MSSA (n) & \%SA & \%MRSA \\
\hline Mouth & 20 & 1 & 11 & 60.0 & 8.3 \\
Ear & 35 & 2 & 27 & 82.9 & 6.9 \\
Eye & 30 & 1 & 18 & 63.3 & 5.3 \\
Face and neck & 22 & 2 & 15 & 77.3 & 11.8 \\
Umbilicus & 33 & 2 & 26 & 84.8 & 7.1 \\
Chest and abdomen & 29 & 1 & 19 & 69.0 & 5.0 \\
Back & 30 & 3 & 18 & 70.0 & 14.3 \\
Perineum and anus & 8 & 0 & 4 & 50.0 & 0.0 \\
Upper limb & 16 & 1 & 11 & 75.0 & 8.3 \\
Lower limb & 17 & 2 & 22 & 82.4 & 14.3 \\
Hand & 30 & 5 & 16 & 90.0 & 18.5 \\
Foot & 23 & 4 & 7 & 87.0 & 5.0 \\
Others & 14 & 0 & 206 & 50.0 & 0.0 \\
Total & 307 & 24 & & 74.9 & 10.4 \\
\hline
\end{tabular}


Table 2. Rates of S. aureus and MRSA infection in the study population.

\begin{tabular}{|c|c|c|c|c|c|c|c|c|c|}
\hline \multirow[b]{2}{*}{ Group } & \multirow{2}{*}{$\begin{array}{l}\text { Participants } \mathrm{n} \\
(\%)\end{array}$} & \multirow[b]{2}{*}{ Male gender n (\%) } & \multirow{2}{*}{$\begin{array}{l}\text { Not infected } \\
n(\%)\end{array}$} & \multirow{2}{*}{$\begin{array}{l}\text { MRSA n } \\
(\%)\end{array}$} & \multirow[b]{2}{*}{ MSSA n (\%) } & \multirow[b]{2}{*}{$\%$ MRSA } & \multicolumn{3}{|c|}{$p$ value } \\
\hline & & & & & & & $\begin{array}{c}\text { MRSA \& } \\
\text { not infected }\end{array}$ & $\begin{array}{l}\text { S. aureus \& } \\
\text { not infected }\end{array}$ & $\begin{array}{c}\text { MSSA \& } \\
\text { MRSA }\end{array}$ \\
\hline $0-28 d$ & $45(14.7)$ & $28(62.2)$ & $13(26.7)$ & $2(4.4)$ & $30(66.7)$ & 6.3 & \multirow{6}{*}{0.028} & \multirow{6}{*}{0.032} & \multirow{6}{*}{0.270} \\
\hline $1-12$ mon & $73(23.8)$ & $40(54.8)$ & $10(15.1)$ & $7(7.6)$ & $56(76.7)$ & 9.5 & & & \\
\hline $1-5 y r$ & $80(26.0)$ & $42(52.5)$ & $23(28.8)$ & $4(5.0)$ & $53(66.3)$ & 7.0 & & & \\
\hline $6-12 \mathrm{yr}$ & $51(16.6)$ & $29(56.9)$ & $10(19.6)$ & $8(15.7)$ & $33(64.7)$ & 19.5 & & & \\
\hline $13-18 \mathrm{yr}$ & $58(18.9)$ & $32(52.5)$ & $21(36.2)$ & $3(5.2)$ & $34(58.6)$ & 8.1 & & & \\
\hline Total & 307 & $171(55.7)$ & $77(25.1)$ & $24(7.8)$ & $206(67.1)$ & 10.4 & & & \\
\hline
\end{tabular}

Table 3. Seasonal distribution of MSSA and MRSA infection.

\begin{tabular}{ccccccc}
\hline \multirow{2}{*}{ Season } & MSSA & MRSA & Not infected & \multicolumn{2}{c}{$p$ value } & \\
\cline { 5 - 7 } & $\mathrm{n}(\%)$ & $\mathrm{n}(\%)$ & $\mathrm{n}(\%)$ & MRSA \& not infected & S. aureus \& not infected & MRSA \& MSSA \\
\hline Spring & $49(67.1)$ & $4(5.5)$ & $20(27.4)$ & & & 0.671 \\
Summer & $70(73.7)$ & $11(11.6)$ & $14(14.7)$ & $\mathbf{0 . 0 4 1}$ & $\mathbf{0 . 0 1 6}$ & \\
Autumn & $53(66.3)$ & $6(7.5)$ & $21(26.2)$ & & & \\
Winter & $34(57.6)$ & $3(5.1)$ & $22(37.3)$ & & & \\
\hline
\end{tabular}

Table 4. Resistant patterns of antibiotics in pediatric patients infected by $S$. aureus.

\begin{tabular}{cccc}
\hline Antibiotics & MSSA $(\mathrm{n}=206)$ & MRSA $(\mathrm{n}=24)$ & $p$ value \\
\hline Penicillin & $198(96.1 \%)$ & $24(100 \%)$ & 1.00 \\
Cefoxitin & 0 & $24(100 \%)$ & 1.00 \\
Chloromycetin & $22(10.7 \%)$ & $11(45.8 \%)$ & $<0.001^{*}$ \\
Erythromycin & $163(79.1 \%)$ & $23(95.8 \%)$ & 0.055 \\
Clindamycin & $37(18.0 \%)$ & $16(66.7 \%)$ & $<0.001^{*}$ \\
Gentamycin & $7(3.4 \%)$ & $8(33.3 \%)$ & $<0.001^{*}$ \\
Rifampicin & $8(3.9 \%)$ & $6(25 \%)$ & $0.001^{*}$ \\
TMP-SMX & $6(2.9 \%)$ & $4(16.7 \%)$ & $0.013^{*}$ \\
Tetracycline & 0 & 0 & - \\
Vancomycin & 0 & 0 & - \\
\hline
\end{tabular}

${ }^{\text {a }}$ Trimethroprim-sulfamethoxazole. ${ }^{*} p<0.05$.

Our results showed that over $80 \%$ of infants had $S$. aureus infection, and about one third of them were newborns. Umbilicus was the most common site of isolation of $S$. aureus in the $<1$ year group. Infants with $S$. aureus infections were predominantly from the villages, where the living conditions were poorer and their families had little knowledge of hygiene and infant care. In addition, breastfeeding had been replaced by formula milk feeding in many infants. This may have a strong affect on their immunity and ability to resist infection. Finally in the more remote areas infants are often born at home without help from nursing or medical staff. In the absence of sterile medical equipments, the umbilicus was often cut using scissors or even a piece of tile which was only washed by hot water, greatly increasing the risk of infection.

There are limited reports of the S. aureus infection rates in pre-school and school-age children with SSTIs.
This study revealed that the infection rate in primary school students (6-12 yr group) was a little lower to that of infants but that the proportion of these infections caused by MRSA was highest. At this age, children play with their fellow students and pay little attention to hygiene, providing perfect conditions for the spread of pathogen bacteria. Many children from more remote areas have to board at the primary schools, which lack many facilities including running water. Other studies also showed that crowded living conditions, including competitive contact sports and child care attendance may contribute to $S$. aureus and particularly MRSA infection [11].

Treatment of uncomplicated SSIs is often by incision and drainage [11], but this is frequently supported by antibiotic therapy especially if living conditions are poor. As the antibiotic resistance increases and patterns of this resistance become more variable, it is necessary for clinicians to be aware of susceptibility patterns in the region in order to use appropriate therapy.

High rates of resistance to erythromycin in this population preclude the use of macrolide therapy for both MSSA and MRSA. These rates were consistent with most studies $[12,13]$. Clindamycin has been shown to be an effective agent in clinical treatment of CA-MRSA in the USA [14], but it appears that local strains were more resistant with over two thirds of MRSA isolates displaying resistance. Studies from New York, Minnesota and Chicago also reported high frequencies $(67 \%-94 \%)$ of clindamycin non-susceptibility which was demonstrated to be inducible resistance $[15,16]$. In our study, about $50 \%$ of MRSA demonstrated inducible clindamycin resistance. Resistance to clindamycin may vary considerably between regions and may be associated with the em- 
pirical use of the drug by local physicians. Use of clindamycin to treat inducible strains has been associated with increased risk of treatment failure [15] and it is suggested that clindamycin use is discontinued if the inducible phenotype is identified locally.

In contrast to European studies which have reported high resistance to TMP-SMX in MRSA varying from $53 \%$ to $76 \%$ [17], resistance to this agent remained fairly low suggesting that it could be an alternative agent in treating uncomplicated SSTIs caused by MRSA. Other antibiotics, including gentamicin and rifampicin had higher resistance rates than elsewhere [18]. This may be linked with the different antibiotic preferences local physicians as well as dissemination of resistant strains, which might increase selection pressure. In addition, antibiotic agents are quite easily available from pharmacies without a prescription allowing for misuse.

\section{CONCLUSION}

With the increased burden of CA-MRSA infection in pediatrics, more attention must be paid to disadvantaged groups. Their specific physical and psychological characteristics and their surroundings may contribute to the spread of CA-MRSA. Functional infection control program should be established to help to reduce incidence of infections. In addition, resistance patterns of CA-MRSA need to be monitored locally to help clinicians choose appropriate empiric antimicrobial agents.

\section{ACKNOWLEDGEMENTS}

We would like to thank Dr. Maureen Boost in the Hong Kong Polytechnic University for reviewing the manuscript.

\section{REFERENCES}

[1] David, M.Z. and Daum, R.S. (2010) Community-associated methicillin-resistant Staphylococcus aureus: Epidemiology and clinical consequences of an emerging epidemic. Clinical Microbiology Reviews, 23, 616-687. http://dx.doi.org/10.1128/CMR.00081-09

[2] Herold, B.C., Immergluck, L.C., Maranan, M.C., Lauderdale, D.S., Gaskin, R.E., et al. (1998) Community-acquired methicillin-resistant Staphylococcus aureus in children with no identified predisposing risk. Journal of the American Medical Association, 279, 593-598. http://dx.doi.org/10.1001/jama.279.8.593

[3] Gosbell, I.B., Mercer, J.L., Neville, S.A., Crone, S.A., Chant, K.G., et al. (2001) Non-multiresistant and multiresistant methicillin-resistant Staphylococcus aureus in community-acquired infections. Medical Journal of Australia, 174, 627-630.

[4] Wu, K.C., Chiu, H.H., Wang, J.H., Lee, N.S., Lin, H.C., et al. (2002) Characteristics of community-acquired methicillin-resistant Staphylococcus aureus in infants and children without known risk factors. Journal of Microbi- ology, Immunology, and Infection, 35, 53-56.

[5] Fergie, J.E. and Purcell, K. (2001) Community-acquired methicillin-resistant Staphylococcus aureus infections in South Texas children. Pediatric Infectious Disease Journal, 20, 860-863. http://dx.doi.org/10.1097/00006454-200109000-00007

[6] Hiramatsu, K., Cui, L., Kuroda, M. and Ito, T. (2001) The emergence and evolution of methicillin-resistant Staphylococcus aureus. Trends in Microbiology, 9, 486-493. http://dx.doi.org/10.1016/S0966-842X(01)02175-8

[7] Del Giudice, P., Bes, M., Hubiche, T., Roudiere, L., Blanc, V., et al. (2011) Clinical manifestations and outcome of skin infections caused by the community-acquired methicillin-resistant Staphylococcus aureus clone ST80-IV. Journal of the European Academy of Dermatology and Venereology, 25, 164-169. http://dx.doi.org/10.1111/j.1468-3083.2010.03731.x

[8] Eady, E.A. and Cove, J.H. (2003) Staphylococcal resistance revisited: Community-acquired methicillin resistant Staphylococcus aureus-An emerging problem for the management of skin and soft tissue infections. Current Opinion in Infectious Diseases, 16, 103-124.

http://dx.doi.org/10.1097/00001432-200304000-00007

[9] Clinical and Laboratory Standards Institute (2010) Performance standards for antimicrobial susceptibility testing: Twentieth informational supplement. Clinical and Laboratory Standards Institute, Wayne.

[10] Fritz, S.A., Garbutt, J., Elward, A., Shannon, W. and Storch, G.A. (2008) Prevalence of and risk factors for community-acquired methicillin-resistant and methicillinsensitive Staphylococcus aureus colonization in children seen in a practice-based research network. Pediatrics, 121, 1090-1098. http://dx.doi.org/10.1542/peds.2007-2104

[11] Ortega-Loayza, A.G., Diamantis, S.A., Gilligan, P. and Morrell, D.S. (2010) Characterization of Staphylococcus aureus cutaneous infections in a pediatric dermatology tertiary health care outpatient facility. Journal of the American Academy of Dermatology, 62, 804-811. http://dx.doi.org/10.1016/j.jaad.2009.07.030

[12] Kaplan, S.L., Hulten, K.G., Gonzalez, B.E., Hammerman, W.A., Lamberth, L., et al., (2005) Three-year surveillance of community-acquired Staphylococcus aureus infections in children. Clinical Infectious Diseases, 40, 1785-1791. http://dx.doi.org/10.1086/430312

[13] Fridkin, S.K., Hageman, J.C., Morrison, M., Sanza, L.T., Como-Sabetti, K., et al. (2005) Methicillin-resistant Staphylococcus aureus disease in three communities. New England Journal of Medicine, 352, 1436-1444. http://dx.doi.org/10.1056/NEJMoa043252

[14] Herigon, J.C., Hersh, A.L., Gerber, J.S., Zaoutis, T.E. and Newland, J.G. (2010) Antibiotic management of Staphylococcus aureus infections in US children's hospitals, 1999-2008. Pediatrics, 125, E1294-E1300. http://dx.doi.org/10.1542/peds.2009-2867

[15] Frank, A.L., Marcinak, J.F., Mangat, P.D., Tjhio, J.T., Kelkar, S., et al. (2002) Clindamycin treatment of methicillin-resistant Staphylococcus aureus infections in children. Pediatric Infectious Disease Journal, 21, 530-534. http://dx.doi.org/10.1097/00006454-200206000-00010 
[16] Martinez-Aguilar, G., Hammerman, W.A., Mason, E.O. and Kaplan, S.L. (2003) Clindamycin treatment of invasive infections caused by community-acquired, methicillin-resistant and methicillin-susceptible Staphylococcus aureus in Children. Pediatric Infectious Disease Journal, 22, 593-598.

http://dx.doi.org/10.1097/01.inf.0000073163.37519.ee

[17] Gemmell, C.G., Edwards, D.I., Fraise, A.P., Gould, F.K., Ridgway, G.L., et al. (2006) Guidelines for the prophylaxis and treatment of methicillin-resistant Staphylococ- cus aureus (MRSA) Infections in the UK. Journal of Antimicrobial Chemotherapy, 57, 589-608.

http://dx.doi.org/10.1093/jac/dk1017

[18] Elsahn, A.F., Yildiz, E.H., Jungkind, D.L., Abdalla, Y.F., Erdurmus, M., et al. (2010) In vitro susceptibility patterns of methicillin-resistant Staphylococcus aureus and coagulase-negative staphylococcus corneal isolates to antibiotics. Cornea, 29, 1131-1135.

http://dx.doi.org/10.1097/ICO.0b013e3181d2ce25 